\title{
Wireless Ice Detection and Monitoring using Flexible UHF RFID Tags
}

\author{
Mahmoud Wagih, Member, IEEE, and Junjie Shi
}

\begin{abstract}
Owing to its low relative permittivity, very few microwave sensors have been developed for monitoring ice deposition. This paper presents the first use of UHF RFID tags for wireless RF ice sensing applications. Despite its low permittivity, the existence of ice as a superstrate on a planar ultra-thin dipole antenna can lower the resonance frequency of the antenna significantly. The RFID tags, having a measured unloaded range of $9.4 \mathrm{~m}$, were evaluated for remotely detecting the formation of ice in various scenarios up to $10 \mathrm{~m}$ from the reader, as well as monitoring the ice thawing, based on the Relative Signal Strength (RSS) in a phase-free approach. In most RSS-based sensing approaches, the tag's read-range is reduced as the RSS decreases in response to the stimulant. However, the proposed sensing mechanism does not reduce the tag's range; the ice superstrate improves the impedance matching of the tags and increases their radar cross section (RCS), resulting in a $10 \mathrm{~m}$ loaded read-range with over $12 \mathrm{~dB}$ ice-sensitivity, in an echoic multi-path environment. The proposed sensing mechanism achieves an accuracy of $86 \%$ for tags positioned between 0.5 and $10.5 \mathrm{~m}$ with various obstructions. When subjected to line-of-sight metallic obstruction, the sensor still maintains $80 \%$ accuracy. The long range and high sensitivity of the proposed sensing mechanics show that UHF RFID is a promising method for detecting the formation of ice in future smart cities.
\end{abstract}

Index Terms-Antennas, RFID, Materials, Relative Permittivity Measurement, Wireless Sensing

\section{INTRODUCTION}

$\mathbf{L}$ ONG-range Ultra High Frequency (UHF) radio frequency identification (RFID) tags have been widely used for a variety of battery-less on-demand sensing applications [1], [2]. The ability to interrogate a varying number of tags and reconstruct information about their surroundings has enabled a variety of applications. For example, localization [3], electricity metering [4], moisture sensing [5]-[7], mechanical deformation sensing [8], structural monitoring [9], in addition to fluids characterization [10] are among the various applications of RFID-based sensing. With UHF RFID readers becoming ubiquitous in various domains, RFID sensing can be considered a low-cost and low-maintenance approach to battery-less wireless sensing. Furthermore, it was previously shown that RFID tags can operate in extremely cold environments as well as under accumulated ice [11].

This work was supported by the UK Engineering and Physical Sciences Research Council (EPSRC) under Grant EP/P010164/1. (Corresponding author: Mahmoud Wagih)

The authors are with the School of Electronics and Computer science, University of Southampton, Southampton, SO17 1BJ, U.K. (email:mahm1m19@soton.ac.uk)

Digital Object Identifier: , datasets used in this paper are available from the University of Southampton repository at DOI: 10.5258/SOTON/D1849
The detection of ice accumulation on various surfaces such as roads, aircrafts, power cables, and pipe-works is of paramount importance. For example, ice formation on wind turbines could lead to up to a $20 \%$ loss in the power production [12], requiring regular helicopter deicing. In addition, in the US alone, over 250,000 households experienced property damage due to freezing water pipes resulting in 10 billion dollars insurance payments over a decade [13]. Moreover, icerelated injuries in a workplace are a main cause of lost work days with over 2,900 ice-caused hospital admissions in the U.K. alone in 2014/15, and over 42,000 work absences in the U.S. [14]. This has motivated research into ice detection mechanisms such as near infrared (NIR) cameras and image processing [15]. While a plethora of RF-based approaches were investigated for remote ice and snow monitoring using GPS signals and satellites [16], the use of wireless RF sensing for ice detection in urban and smart cities applications has not been reported.

Despite its ubiquity in a variety of applications, UHF RFID sensing has not been applied for detecting ice accumulation or deposition. This is attributed to the very low permittivity of frozen particles compared to their liquid form, where the immobility of charge carriers reduces the ionic conductivity of water leading to ice being commonly described as being "almost transparent to microwaves" [17]. Recently, a two-port microstrip resonator, implemented on a low-loss RF PCB, was proposed for freeze and thaw detection [18]. A ground plane-integrated heater was later proposed based on the same resonator showing a solution for detecting and removing the ice [19]. However, owing to the resonator design, the sensors, [18], [19] have a high (3.5-4.5 GHz) frequency of operation compared to widely available commercial transceivers and readers such as sub-1 GHz RFID ICs/readers and $2.4 \mathrm{GHz}$ Bluetooth/Zigbee transceivers. A low-frequency capacitive sensor was also proposed for ice detection [20]. However, the sensor still requires active sampling circuitry, adding to the cost, complexity and maintenance overhead of the approach. Therefore, a need exists for a wireless, compact, battery/maintenance-free and low-cost solution enabling the detection of ice.

In this paper, the antenna-based ice detection is proposed, and implemented using UHF RFID tags for wireless ice detection and sensing applications. This approach could be demonstrated using different antenna designs based on the application. The key novel findings in this work can be summarized as:

1) Demonstrating the first use of wireless RF sensing for ice detection and low-permittivity stimulants; 
2) Relying on an increase in the tag's relative signal strength (RSS) to detect the stimulant for improved sensitivity without sacrificing the read-range;

In Section II, the RF ice sensing phenomenon is introduced through the simulated antenna parameters. Section III presents the experimental setup including the antenna and reader design, as well as the example test use-cases, with the measured results and discussion in Section IV. The accuracy of the proposed sensing methodology is evaluated in Section V.

\section{RFID ICE SENSING In SMART Cities}

\section{A. RFID Antennas as Ice Sensors}

Ice possesses one of the lowest relative permittivities $\left(\epsilon_{r}=3.2\right)$ and dissipation factors $(\tan \delta=0.0009)$ compared to other stimulants such as moisture [6], or food products [17]. Therefore, for an RF sensor to detect the presence and the formation of thin ice layers, the RF resonator needs to be highly sensitivity to very small changes on its surface.

Most of the reported magnitude-based RFID sensing tags detect the stimulant through a reduction in the RSS, i.e. through detuning or additional losses in the tag [5]. This will result in a reduced read-range when the tag is measured under loading, reducing its applicability for remote wireless sensing. Recently, a tag was shown operating with an $8 \mathrm{~m}$-range at $868 \mathrm{MHz}$, despite being tuned for $915 \mathrm{MHz}$ and having an $S_{11}$ around $-2 \mathrm{~dB}$ [21]. Thus, it is possible to operate the sensing tag at a lower frequency than its unloaded resonance while maintaining a medium read-range. When loaded with the stimulant, the antenna's resonance will shift to a lower frequency resulting in an increase in the RSS in response to the stimulant, owing to an increase in the RFID antenna's realized gain.

To verify the anticipated shift in an antenna's resonance in response to ice-loading, a loop-fed dipole antenna, discussed in detail in the next section, was simulated in CST Microwave Studio (frequency domain solver with $0.2 \mathrm{~mm}$ maximum mesh step) for varying ice thicknesses. The thin ice layer added on the antenna model is $21 \times 9 \mathrm{~cm}$ and variable thickness $t$, with $\epsilon_{r}=3.2$ and $\tan \delta=0.0009$ based on the microwave properties measured in [17]. Fig. 1 shows the simulated input impedance of the antenna for different ice thicknesses $t$.

From Fig. 2, it can be verified that the $75 \mu$ m-thick dipole exhibits a high sensitivity to sub-mm thick ice-loading. Furthermore, the resonance shift to a lower frequency not only improves the impedance matching in the $868 \mathrm{MHz}$ band for a fixed- $Z$ source but also improves the antenna's radiation efficiency. To explain, the additional low-loss superstrate, i.e. ice, increases the antenna's electrical size increasing its radiation resistance and subsequently efficiency. The antenna's far-fields were simulated for varying $t$ to investigate the ice influence on the radiation properties. Fig. 2 shows the increase in the antenna's radiation efficiency (exclusive of mismatch) at $850 \mathrm{MHz}$ under ice-loading.

While the additional ice superstrate results in up to $2 \mathrm{~dB}$ increase in the radiation efficiency (from $80 \%$ to $99 \%$ in
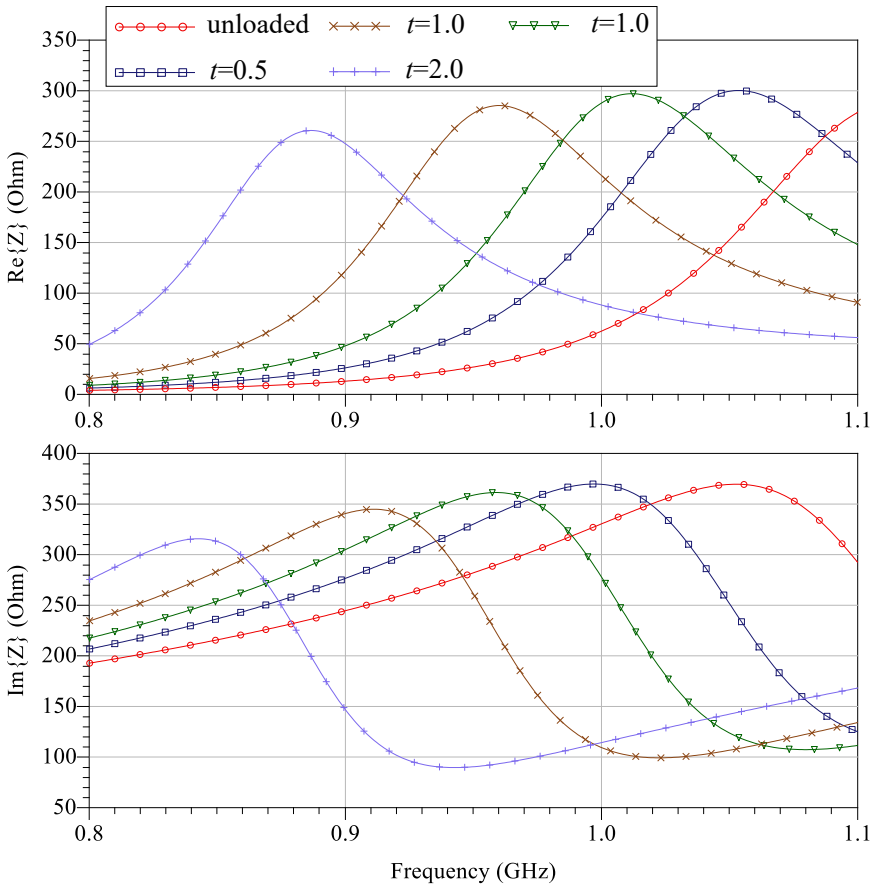

Fig. 1. Simulated input impedance of the RFID antenna under varying thickness ice loading.

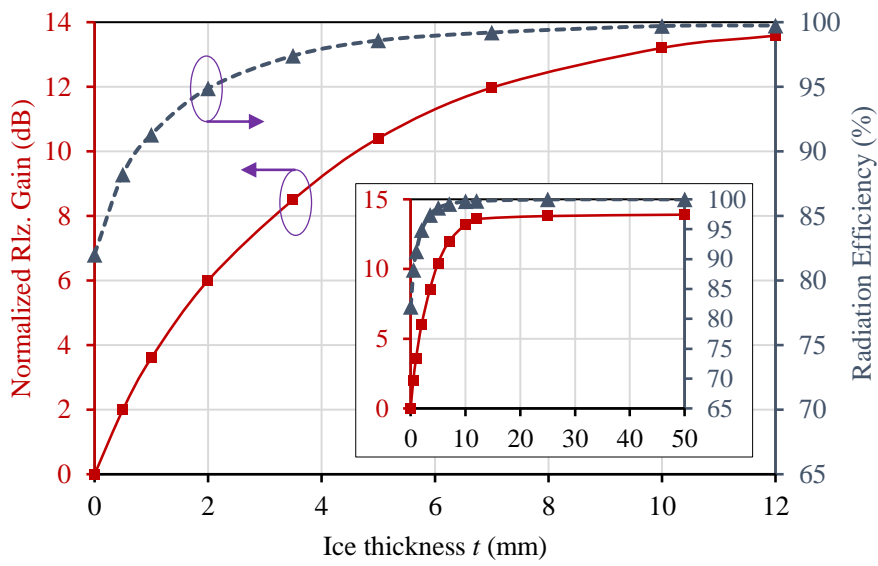

Fig. 2. Simulated realized gain and radiation efficiency of the RFID antenna as a function of the ice superstrate thickness; inset shows the response for $t>12 \mathrm{~mm}$

Fig. 2), the impedance matching effect has the most significant influence on the sensory response. This is observed in the antenna's realized gain, normalized to the unloaded antenna's gain, showing an improvement of over $10 \mathrm{~dB}$ for $5 \mathrm{~mm}$ iceloading. Therefore, the simulated results show that an antenna can act as a highly sensitive detector for the formation of ice, with a response that could be detected wirelessly using an off-the-shelf receiver, e.g. an RFID reader. For ice thicknesses in excess of $12 \mathrm{~mm}$, shown in the inset of Fig. 2, it can be observed that the gain of the tag stabilizes, implying a stable radar cross section (RCS) and translating to no further changes in the detected RSS. Therefore, build-up of thick ice does not interfere with the operation of a tag as an ice-detection sensor.

The read-range of an RFID tag can be estimated analytically 
based on the free space path loss using

$$
\text { Read range }=\frac{\lambda}{4 \pi} \sqrt{\frac{P_{t} G_{t} G_{r}}{P_{t h}} \times\left(1-\left|S_{11}\right|^{2}\right)},
$$

where $G_{t}$ and $G_{r}$ are the transmitter (reader) and receiver (tag) antenna gains, respectively, $P_{t h}$ is the RFID IC's sensitivity, and $P_{t}$ is the transmitter power [22]. The improved matching of the antenna (lower $S_{11}$ ) will allow the loaded tag's range to exceed its unloaded counterpart, preserving its range in a realistic lossy multi-path environment. When the ID is backscattered, the RSS is given by

$$
\mathrm{RSS}=\Gamma_{\text {Ref. }} P_{t} G_{t}^{2} G_{r}^{2}\left(\frac{\lambda}{4 \pi d}\right)^{4}
$$

where $\Gamma_{\text {Ref. }}$ is the reflection coefficient when the antenna is shorted for modulation, and $d$ is the distance between the reader and the tag, assuming an ideal and linear switching response by the RFID IC. Therefore, the increase in the tag's gain $G_{r}$ will be directly detected at the reader through the RSS, provided that $P_{\mathrm{RX}}>P_{t h}$ for the tag to turn-on. As the reader antenna is often circularly polarized while the tag is linearly polarized, $G_{\mathrm{dBc}}$ to $G_{\mathrm{dBi}}$ will incur a $3 \mathrm{~dB}(50 \%)$ loss. The circular to linear polarization loss is incurred when transmitting information and power tag, and when the ID is backscattered to the reader, resulting in a $6 \mathrm{~dB}$ loss in the detected RSS. However, by using a linearly-polarized tga with a circularly-polarized reader, the tag's response becomes consistent irrespective of its angular alignment with the reader [23], [24].

\section{B. Example RFID Ice Sensing System and Application}

In locations where ice build-up could cause damage or injury, such as water pipes, steps, and wind turbines, RFID ice-sensing tags can be deployed. Owing to their ability to conform to different surfaces, RFID tags implemented on flexible and thin substrates are required. UHF RFID readers can then be deployed to create various coverage "cells", as shown in Fig. 3. To improve the coverage, long read-range tags are essential, reducing the number of readers required to cover a certain area. To explain, based on an RFID tag read-range of a $10 \mathrm{~m}$, a reader based on a microstrip patch antenna with a $60^{\circ}$ half-power beamwidth can cover an area over $38 \mathrm{~m}^{2}$. Moreover, the tags need to be packaged using thin and conformable packaging, yet be mechanically robust and waterproof to withstand deployment around a city. Similarly, the thickness of the substrate and the encapsulating superstrate needs to be minimized, to enhance the antenna's sensitivity to thin ice layers.

Following deployment, the RSS of the unloaded tags can be measured before any frost or ice forms. The measured $\mathrm{RSS}_{\text {Unloaded }}$ will primarily be a function of the channel gain $G_{C}$ between the reader and the tag, comprising the effects of distance, multi-path reflections, and permanent obstructions. Based on Fig. 2, the presence of ice, $t>0 \mathrm{~mm}$, will increase the effective gain and RCS of the tags. The presence of ice can then be defined as a binary variable $p$, which influences

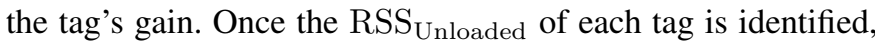
the RFID readers can then interrogate the tags periodically to
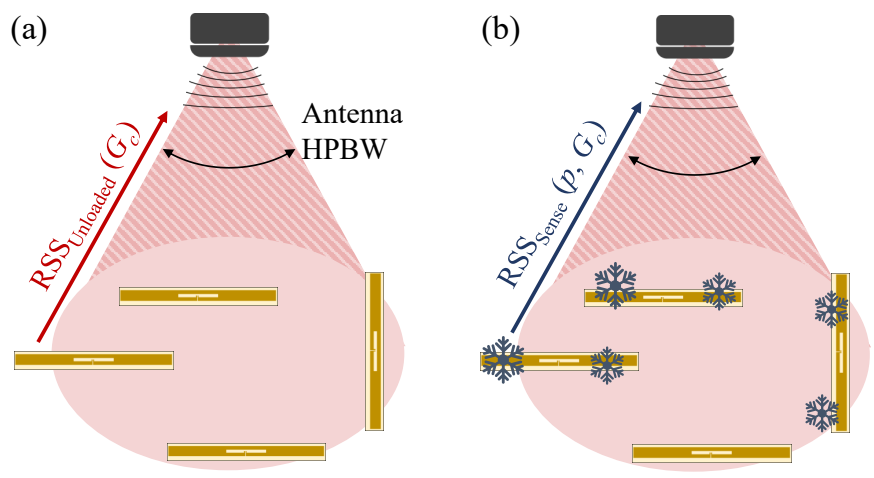

Fig. 3. RFID ice sensors deployment: (a) unloaded RSS measurement to determine the baseline RSS; (b) sensor interrogation.

measure their sensory response $\mathrm{RSS}_{\text {Sense }}$. Subsequently the change in the RSS can be evaluated for individual tags as $\Delta \mathrm{RSS}=\mathrm{RSS}_{\text {Sense }}-\mathrm{RSS}_{\text {Unloaded }}$. The output of the sensing system, i.e. the presence of ice $p$, can be evaluated based on

$$
\Delta \mathrm{RSS}>\mathrm{RSS}_{\text {Threshold }} \rightarrow p=1,
$$

where $\mathrm{RSS}_{\text {Threshold }}$ is defined as the minimum change in the RSS caused by the presence of ice on the RFID sensing tags, to maximize the sensitivity of the proposed sensing approach. The experimental characterization of the tags' detailed in Sections III and IV endeavors to identify $\mathrm{RSS}_{\text {Threshold }}$ across various real-world use-cases, where the accuracy of the ice detection is evaluated in Section V.

RSS is chosen as the sensing parameter as it is highly influenced by the presence of ice $p$, which affects the antenna's gain and RCS. However, other parameters such as the phase of the backscattered signal could be used or the resonant frequency of the tags. Nevertheless, phase does require more complex readers whereas all commercially-available Gen-2 RFID readers can detect the RSS. The resonant frequency of the tag could be used for detecting the presence of ice, by interrogating the tag over the full $850-950 \mathrm{MHz}$ bandwidth [25]. Nevertheless, in most countries, RFID readers are only allowed to operate in specific channels in either the 868 (868$870 \mathrm{MHz}$ ) or $915 \mathrm{MHz}(902-915 \mathrm{MHz})$ license-free bands, where the rest of the $850-950 \mathrm{MHz}$ spectrum is allocated to licensed applications such as GSM. Therefore, the proposed sensing approach, based entirely on the RSS, is the most scalable based on existing wireless standards and commerciallyavailable readers.

\section{EXPERIMENTAL SETUP AND TAG CHARACTERIZATION}

\section{A. Antenna Design and Characteristics}

The RFID tags used in this work are based on thin and flexible dipole antennas with an inductive tuning loop. The antenna is designed to directly match the RFID IC with a complex impedance and is fabricated using standard photolithography. The tags are fully encapsulated using vacuum-formed polyimide to improve their robustness and waterproofing, in order to withstand deployment in a smart city environment. The details of the antenna tuning, in addition to the tag fabrication and encapsulation method are detailed in [21]. For improved ice-sensitivity, the antenna needs to be implemented on a thin 


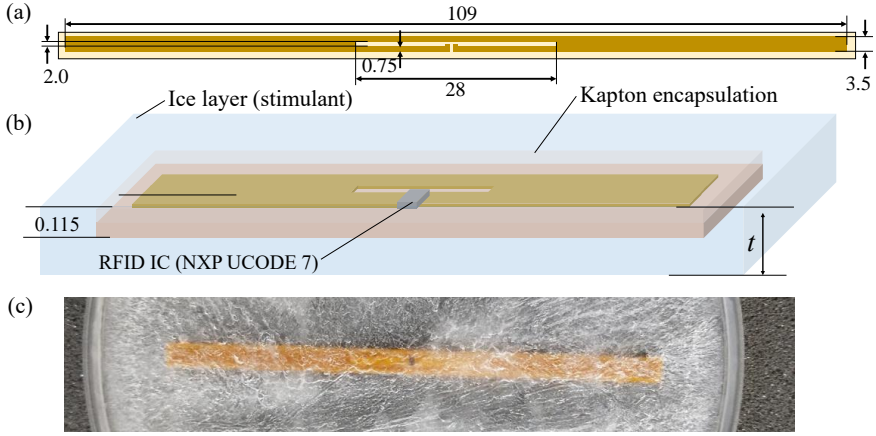

Fig. 4. The ice-sensing RFID tag: (a) the dimensions (in $\mathrm{mm}$ ) of the antenna; (b) 3D layout and dimensions of the encapsulated RFID tag inside the ice loading; (c) photograph of the tag in ice.

substrate, to be able to detect the presence of thin ice sheets $(t=1 \mathrm{~mm})$, as shown in Fig. 2.

Fig. 4-a and $b$ shows the layout and dimensions of the antenna used in this work. The RFID Gen-2 IC is the NXP U-CODE 7. Having a $-21 \mathrm{dBm}$ read-sensitivity, the expected read-range is up to $15 \mathrm{~m}$ from a reader equivalent isotropic radiated power (EIRP) of $33 \mathrm{dBm}$ and a $0 \mathrm{dBi}$ tag antenna using based on the free space path loss (FSPL). Unloaded, the RFID tags achieve a measured read-range of 9.4 metres at 860 $\mathrm{MHz}$, where their optimum matching to the datasheet's input impedance is at $915 \mathrm{MHz}$. The tags were only characterized around $868 \mathrm{MHz}$ due to the EU reader frequency limits.

\section{B. RFID Ice-Sensing Test Setups}

The proposed RFID-based sensors are characterized to investigate their performance in three use-cases: (a) detecting the formation of ice during the freezing process; (b) remotely detecting the existence of an ice layer on the sensing tag in a variety of environments and read-ranges; (c) remotely observing thawing ice. This section describes the test setups used to evaluate the tags for these use-cases.

A commercial hand-held Gen-2 RFID reader (Zebra RFD$8500)$ is used to interrogate the tags. The reader has an equivalent isotropic radiated power (EIRP) of $3 \mathrm{~W}$ and operates in the EU $868 \mathrm{MHz}$ license-free band. Connected to a smartphone via Bluetooth, the reader reports the RSS of up to 200 tags with a $1 \mathrm{~dB}$ resolution. By operating at a single frequency and relying entirely on the RSS for sensing, the proposed RFID-based ice sensor is compliant with regional frequency regulations where the stimulant can be detected without the need for a frequency sweep spanning the full bandwidth between 860 and $940 \mathrm{MHz}$, often found in several RFID sensing applications [25].

To investigate the tags' freezing response, the RFID tags were placed inside a TEFCOLD SE10-45 freezer at $-20^{\circ} \mathrm{C}$. The reader was placed outside the freezer to interrogate the tags intermittently. Due to the high RF shielding caused by the freezer's walls, and the additional reflections inside the freezer, the measurements were performed at $50 \mathrm{~cm}$ from the freezer, with $10 \mathrm{~cm}$ between the tag and the freezer's inner wall.
TABLE I

ICE-LOADED RFID TAG TEST SETUPS.

\begin{tabular}{|l|l|l|l|}
\hline & Test setup & $\begin{array}{l}\text { Unloaded } \\
\text { RSS } \\
(\mathbf{d B m})\end{array}$ & $\begin{array}{l}\text { Loaded } \\
\text { RSS } \\
(\mathbf{d B m})\end{array}$ \\
\hline \hline $\mathrm{A}$ & $\begin{array}{l}\text { Indoor, } d=7.4 \mathrm{~m} \text { and tag/reader } \\
h=1.85 \text { (Fig. 6-a) }\end{array}$ & -70 & -62.5 \\
\hline $\mathrm{B}$ & $\begin{array}{l}\text { Indoor, } D=5.1, \text { reader } h=1.85 \text { and } \\
\text { ag on-ground (Fig. 6-b) }\end{array}$ & -67.7 & -57.7 \\
\hline $\mathrm{C}$ & $\begin{array}{l}\text { Indoor, d=7.7, reader/tag } h=1.7 \\
\text { (Fig. 6-d) }\end{array}$ & -57 & -53 \\
\hline $\mathrm{D}$ & $\begin{array}{l}\text { Outdoor, d=6.5 m and tag/reader } \\
h=0.8 \text { (Fig. 6-e) }\end{array}$ & -61 & -57 \\
\hline
\end{tabular}

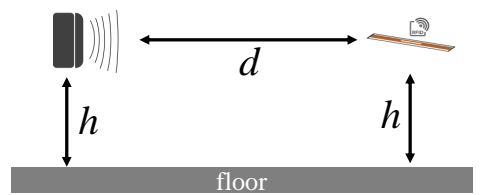

(a)

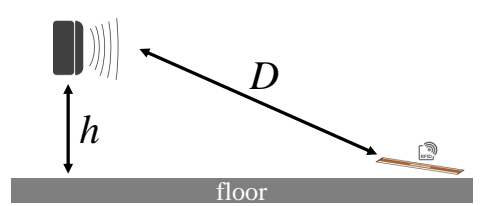

(b)

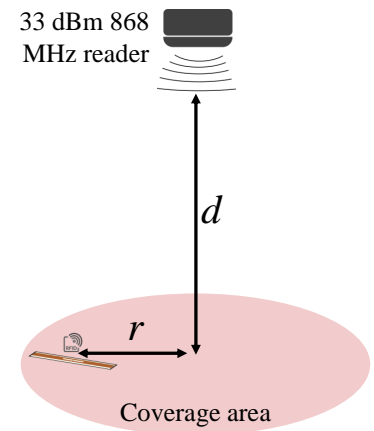

(c)
Fig. 5. Measurements setups of the RFID ice sensor: (a) tag and reader elevated at height $h$; (b) elevated reader and on-ground tag; (c) vertical offground reader and on-ground tag.

Following the formation of full ice layer on the tag's surface, the input impedance of the antenna is measured using a two-port Rohde and Schwarz ZVB4 VNA to understand the ice influence on the tag's matching, and for validating the simulated results in Section I. The differential input impedance was measured using a balanced coaxial jig connected to the VNA's imbalanced ports using a widely known method detailed in [26], widely used for complex impedance antennas for RFID and Schottky-base rectennas [27]. The balanced jig is soldered on the tag prior to freezing with the coaxial connector protruding from the ice to enable VNA measurements postfreezing.

The ice-loaded RFID tags were then interrogated in various test conditions to show that the change between the unloaded and loaded tag RSS ( $\triangle$ RSS) is maintained in real-world usecases, summarized in Table I. Fig. 5 shows the measurement setups and separations of the ice-loaded RFID tags. The photographs of selected test setups are shown in Fig. 6.

The final test carried out is to assess the sensor's response for detecting thawing events. The water content in thawing ice will significantly lower the resonant frequency due to the high permittivity of water $\left(\epsilon_{r}=78\right)$ compared to ice [17], detuning the tags. Furthermore, as water has a higher $\tan \delta$ of 0.16 , the radiation efficiency of the tags will degrade.

Multiple tags with varying ice thicknesses are then used to asses the tags' sensitivity to thawing. The ice-loaded tags are left at room temperature $\left(24^{\circ} \mathrm{C}\right)$ where they were interrogated periodically every $30 \mathrm{~s}$. The measurements were performed using the setup in Fig. 5-a, at $d=3 \mathrm{~m}$. The experimental results 

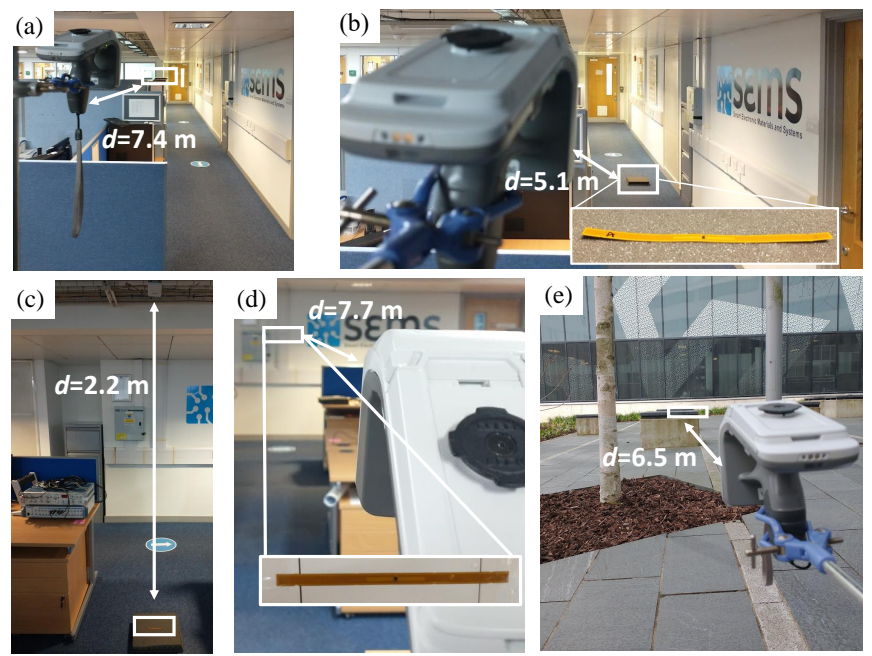

Fig. 6. Photographs of the antenna measurement setups: (a) elevated reader and tag from Fig. 5-a; (b) on-ground tag from Fig. 5-b; (c) ceiling reader from Fig. 5-c; (d) wall-mounted tag; (e) outdoor measurements at $d=6.5 \mathrm{~m}$ using an off-ground setup (Fig. 5-a).

of the fore-mentioned test setups are presented and discussed in the next section.

\section{Measurement Results and Discussion}

\section{A. Ice-Formation and Detection}

The ice-loaded RFID antenna was characterized following a complete freeze. The ice thickness was measured to be approximately $7 \mathrm{~mm}$ over the antenna's surface. The antenna's input impedance, measured using a coaxial jig connected to the twoport VNA is shown in Fig. 7, before and after the antenna is covered by ice. The resonance shift of approximately $200 \mathrm{MHz}$ $\left(0.79 \times f_{0}\right)$ caused by the ice superstrate shows a very close agreement between the CST simulation and measurements. The slight discrepancy observed in the ice-loaded case is attributed to variations between the uniform rectangular ice layer in simulation, and the tapered ice layer shown in the inset in Fig. 7, in addition to the possible impurities in the tap water resulting in a higher $\tan \delta$. Nevertheless, the observed sensory response will result in improving the realized gain and the radiation efficiency $\eta_{\text {Rad. }}$, given by

$$
\eta_{\text {Rad. }}=\frac{R_{\text {Rad. }}}{R_{\text {Rad. }}+R_{\text {Loss }}}
$$

for a wire antenna where $R_{\text {Rad. }}$ and $R_{\text {Loss }}$ are the radiation and loss resistances, respectively. Therefore, the increase in the radiation resistance $R_{\text {Rad. }}$ at resonance maximizes the

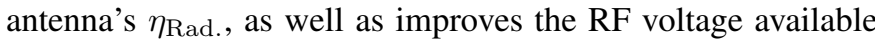
at the RFID rectifier's input [28]. In addition, the higher $\Re\{Z\}$ will increase the observed RSS, owing to a larger change in the reflection coefficient between the short circuit condition (introduced by backscattering) and the antenna-IC matched case.

Observing the measured RSS in Table I, it can be observed that once the ice layer is formed on the antenna, the RSS exhibits a clear change of at least $4 \mathrm{~dB}$. Therefore, the approach of detecting ice presence through an increase in the

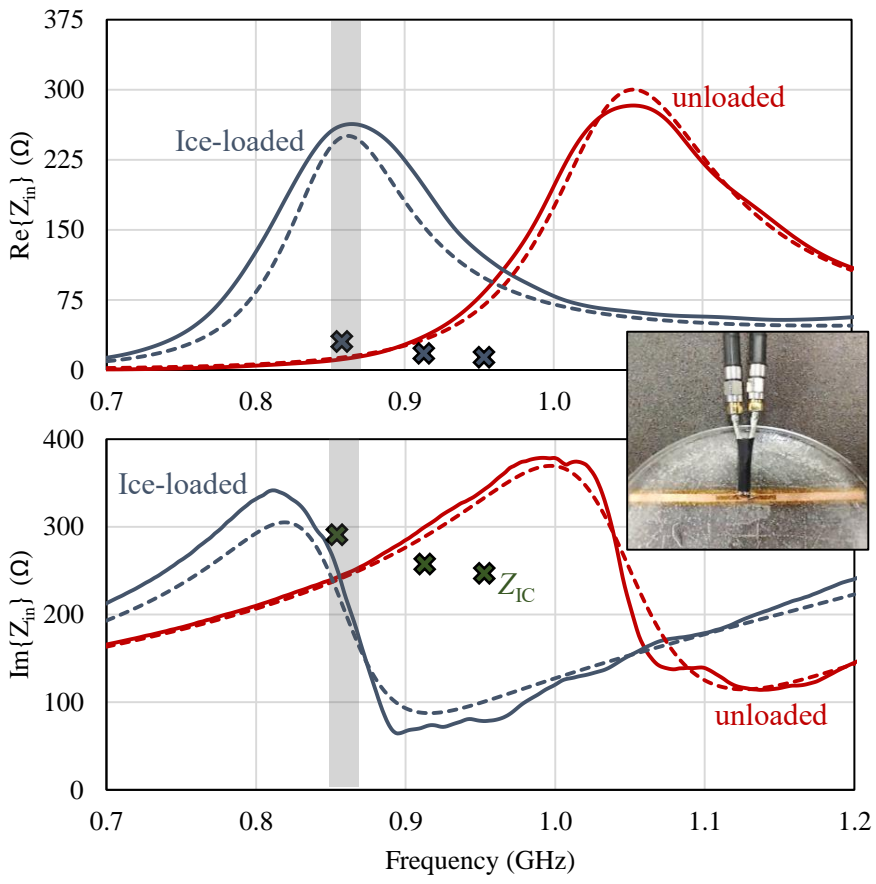

Fig. 7. Simulated (dashed) and measured (solid) $Z_{\text {in }}$ before and after $7 \mathrm{~mm}$ thick ice-loading; inset shows the loaded measurement setup.

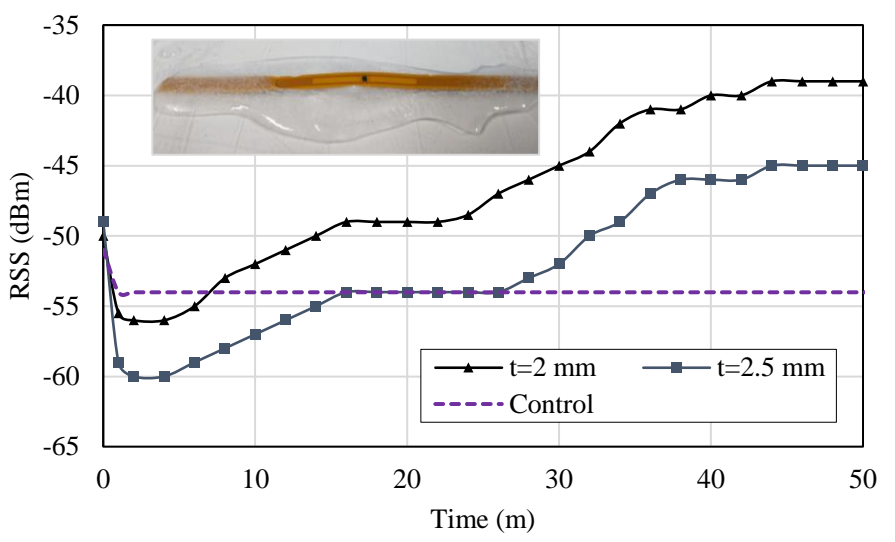

Fig. 8. Measured RSS of the freezing tags with water-droplets loading inside a $-20^{\circ} \mathrm{C}$ freezer; inset shows the non-uniform $t=2.5$ ice layer on the tag.

tag's RSS is validated. For example, in case C, the antenna was mounted on a plastic surface acting as a thick substrate, shown in Fig. 6-d, which could mask the ice effect. However, the measured RSS shows that the presence of ice can still be detected in spite of the added thick plastic substrate.

\section{B. Ice Detection and Sensing Read-Range}

The measured RSS of the tags placed inside the freezer is shown in Fig. 8. Water droplets were added on top of the tagunder-test to mimic dew prior to frost formation. An additional tag was measured inside the freezer without humidity or added water droplets, shown as the control RSS in Fig. 8.

After about 2 minutes at $-20^{\circ} \mathrm{C}$, the control tag suffers from a $4 \mathrm{~dB}$ decline in the RSS, this is attributed to the temperature effect on the RFID IC, where a low temperature results in variations in the transistors threshold voltage [29], increasing the 


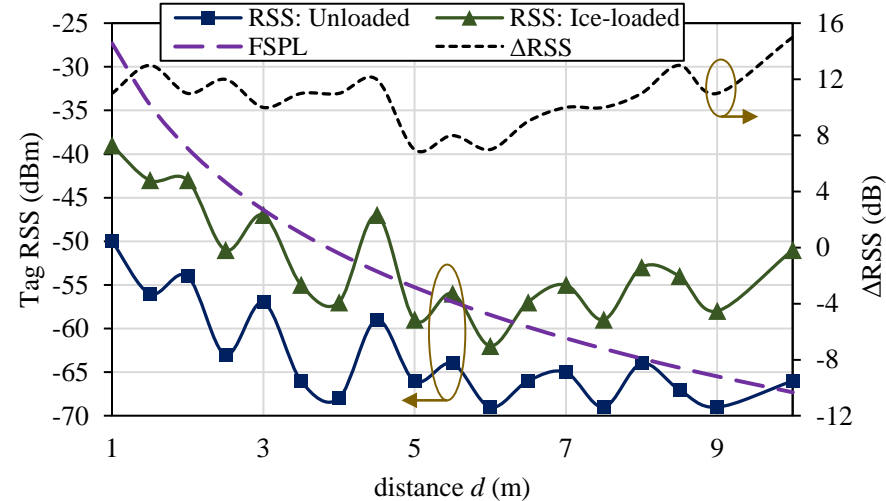

Fig. 9. Measured RSS of the sensor tags before and after ice loading for varying distance (setup-a).

parasitic losses inside the tag's rectifier and modulating switch. However, as the unloaded tag's RSS stabilizes at $-54 \mathrm{dBm}$, it can be confirmed that the influence of temperature does not interfere with the tags ability to detect the presence of ice, as observed in the two ice-loaded samples, where their $\triangle$ RSS exceeds $6 \mathrm{~dB}$, and stabilizes after the water has frozen completely in about 45 minutes.

For the icing tags, initial sharp decline in the RSS is attributed to both the temperature effect, observed on the dry control sample, and the high $\tan \delta$ of water. As ice forms, the RSS improves as previously observed in Table I, explained by the measured input impedance shown in Fig. 7 and the sensing phenomenon introduced in Section I.

Following the demonstration of the tag's sensing capability through the VNA $Z$ measurements and the RSS measurements inside a freezer, Fig. 9 shows the measured RSS of the loaded and unloaded tags at varying separations $d$, from a reader at the same height $h=1.85$ (Fig. I-a). The measurements were performed indoors as in Fig. 6-a, to demonstrate the sensor's response in a highly echoic environment. Several application domains such as monitoring pipe-works, electric transformers, and staircases in public spaces will expect the RFID tags to operate in confined indoor spaces, where multi-path effects are more omnipresent than outdoor environments or anechoic chamber lab demonstrations.

From Fig. 9, it can be observed that the tags maintain at least $6 \mathrm{~dB} \triangle \mathrm{RSS}$ sensitivity. At $10 \mathrm{~m}$ from the reader, the tag's $\triangle$ RSS is $14 \mathrm{~dB}$, showing that the proposed sensor can be used at a longer range than the tag's unloaded read-range. This highlights the advantage of designing the tags to improve their $Z$ matching under loading. The observed increase in the RSS at $4.5 \mathrm{~m}$ is attributed to the interference caused by multipath reflections, as the test was carried out in an echoic indoor environment. However, it can be observed from $\triangle$ RSS that the tag maintains its high sensitivity and the presence of ice can still be detected regardless of the multi-path reflections. It is also observed that the measured RSS follows the expected $1 / d^{2}$ relation caused the by the FSPL, calculated using (2) for EIRP $=33 \mathrm{dBm}$ and $G_{r}=2.1 \mathrm{dBi}$. the analytical RSS calculated based on (2), in Fig. 9 is inclusive of the $6 \mathrm{~dB}$ circular-linearcircular polarization mismatch loss. As for $\Delta$ RSS, the 8-14 dB

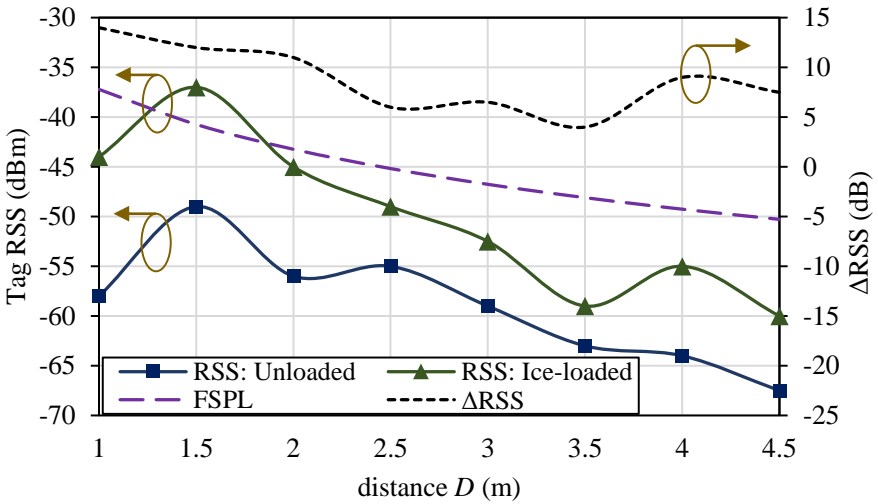

Fig. 10. Measured RSS of the sensor tags before and after ice loading for varying distance where the tag is placed on the ground and interrogated by a reader at $h=1.2$ (setup-b).

RSS gain agrees with the simulated increase in the antenna's gain, in Fig. 2, in response to the $9 \mathrm{~mm}$-thick ice layer.

As ice forms a major slip hazard [14], the tags sensory response when place on the ground is important for public safety applications. Fig. 10 shows the measured RSS for varying distance $D$, based on the setup in Fig. 5-b and 6b. From the measured results, it can be observed that the tag's read-range is reduced to $4.5 \mathrm{~m}$, owing to the additional destructive interference between the transmitted signals from the reader. Nevertheless, the ice-sensitivity still exceeds $4 \mathrm{~dB}$ at all distances considered, with the measured RSS following closely the analytical RSS based on the FSPL from (2). Therefore, a handheld reader, for example carried by a maintenance personnel, can detect the formation of ice on the ground with an RFID sensor up to $4.5 \mathrm{~m}$ away.

Similarly, a ceiling-mounted reader can be used to cover a circular spot whose size is controlled by the antenna's main lobe beamwidth. The RSS results for the ceiling mounted reader (Fig. 5-c) are shown in Fig. 11. This setup resembles a highly-likely deployment scenario for monitoring surfaces such as pavements or platforms with the readers mounted on light-posts or shelters. $d \approx r=2.2 \mathrm{~m}$, the tags maintain a very consistent and high $\Delta$ RSS between 12 and $16 \mathrm{~dB}$. For $r>d$, the tags were not readable (both loaded and unloaded) which is attributed to the relatively more directional radiation pattern of the reader's antenna, which typically maintain a half-power $(-3 \mathrm{~dB})$ beamwidth of $60^{\circ}$, due to using a broadside patch antenna.

The final test carried out is for assessing the sensor's response for thawing detection and monitoring. Fig. 12 shows the measured tag RSS for four different ice thicknesses at 3 metres from the reader. It can be observed that as the iceloaded tags thaw, the increase in the water content in the ice superstrate results in a reduction in the RSS and eventually the reader failing to detect the tag. This is attributed to water's properties $\left(\epsilon_{r}=78 ; \tan \delta=0.16\right)$ resulting in a significant shift in the antenna's resonance. Moreover, the high $\tan \delta$ will result in a reduced radiation efficiency due to an increase in the power absorbed by the water. Therefore, an RFID tag can only be read during the water-ice/ice-water transition stages when the water content is not sufficient to detune the tags. 


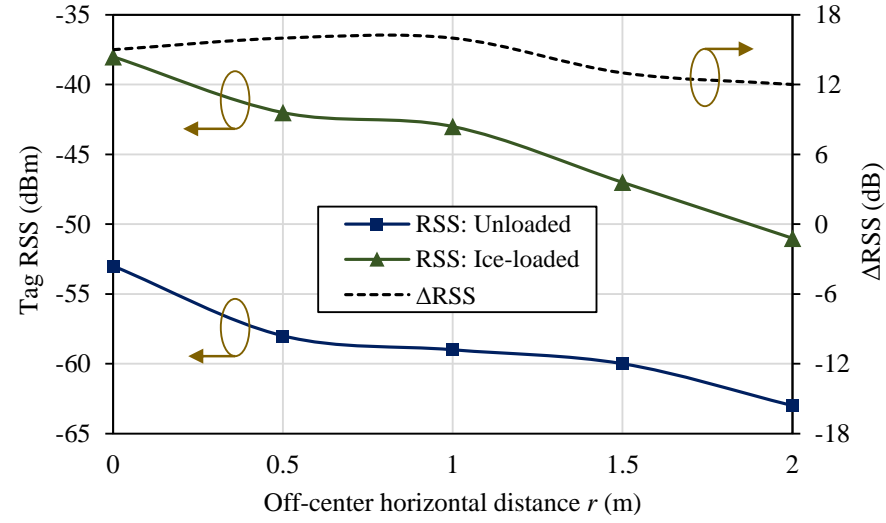

Fig. 11. Measured RSS of the sensor tags before and after ice loading for varying off-axis radii from a ceiling receiver at $d=2.2$ (setup-c).

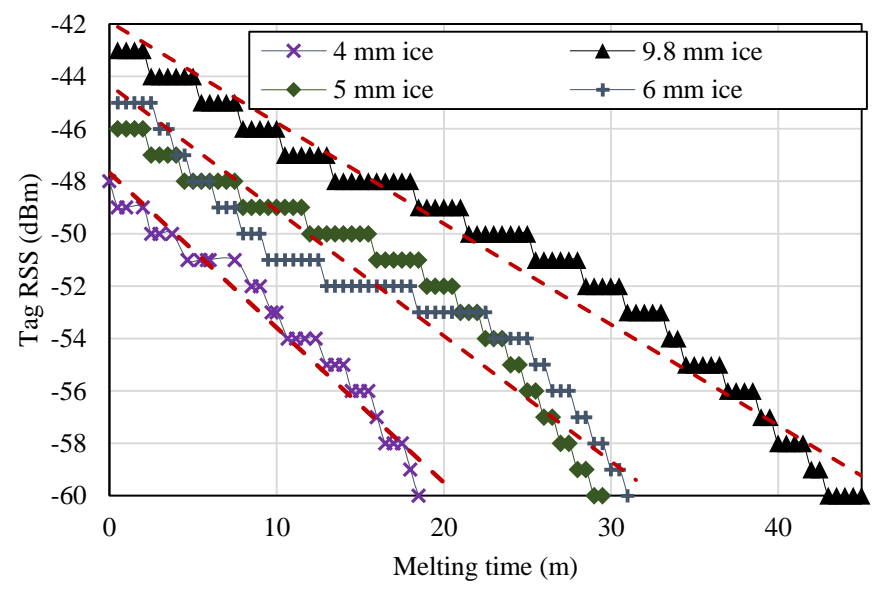

Fig. 12. Measured RSS of the sensor tags during thawing at $24^{\circ} \mathrm{C}$ for different ice thicknesses (setup-a).

For all ice thicknesses investigated $(4-9.8 \mathrm{~mm})$, the melting response is highly linear, evidenced by the close agreement of the best-fit line having an $\mathrm{R}^{2}=0.97$ and 0.95 , for $t=4$ and $9.8 \mathrm{~mm}$, respectively. In addition, the measured RSS before melting (at time $=0$ ) validates the direct proportionality of the antenna's gain (observed through the RSS) to the ice thickness introduced in Section I. Therefore, the proposed RFID sensor is not only capable of detecting ice-formation over a long range in various scenarios, but also capable of observing ice thawing, whose applications extend beyond industrial and smart city sensors to remote and environmental monitoring.

Fig. 12 also shows a correlation between the thickness of the ice sheet and the RSS of the tag, where a higher RSS is observed for thicker ice. For instance, at the 10 minutes mark, the RSS is $-53,-51,-49$, and $-46 \mathrm{dBm}$, for 4,5 , 6 , and $9.8 \mathrm{~mm}$ of ice, respectively. This is in line with the numerically simulated response of the RFID antenna, shown in Fig. 2, where the change in the antenna's gain was a function of the thickness of the ice. The cross-over between the RSS of the tags loaded with 4 and $5 \mathrm{~mm}$-thick ice is attributed to the non-uniform thickness of the ice cover. (a)

(b)
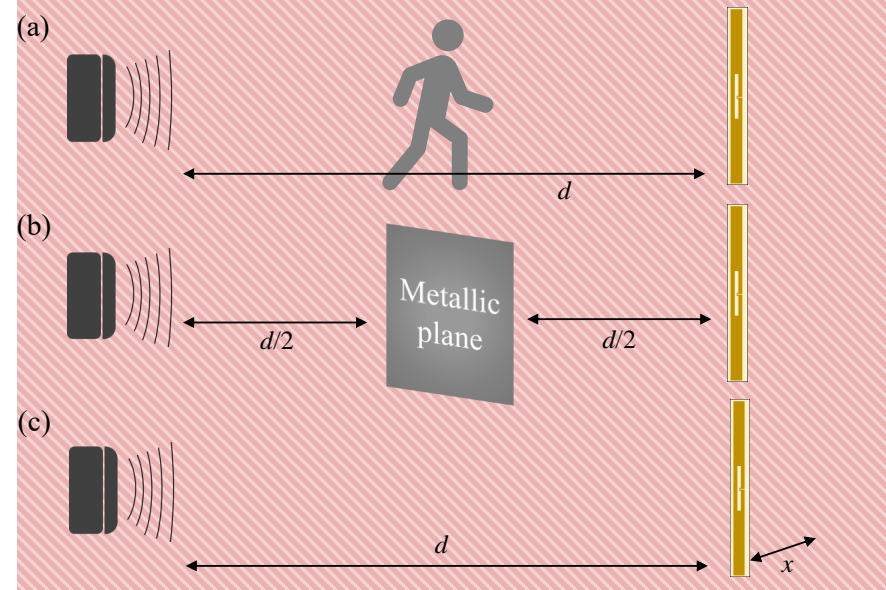

Fig. 13. Measurement setups of the sensor for evaluating its resilience to interference: (a) person blocking; (b) $1 \times 1 \mathrm{~m}$ metal sheet blocking; (c) $1 \times 1 \mathrm{~m}$ metal sheet tag-backing in the near-field.

\section{Sensor Performance Evaluation}

\section{A. RFID Ice Detection}

The sensing strategy introduced in Section I has been evaluated experimentally following the tags' characterization. Based on the minimum $\triangle$ RSS observed in all test setups in Section IV, $\mathrm{RSS}_{\text {threshold }}$ from (3) is set to $4 \mathrm{~dB}$. To evaluate the sensor's performance, loaded and unloaded RSS measurements have been performed in two locations for $0.5<d<10.5 \mathrm{~m}$, based on the maximum read range of the tags. Additional obstructions and interference sources were then added to investigate the accurate detection rate, as well as the false detections caused by external interference, e.g. RF reflection off a moving surface.

First of all, to investigate the performance of an individual tag, as well as its resilience to external interference. The tag was placed at $5 \mathrm{~m}$ from the reader at $h=1.5 \mathrm{~m}$ (Fig. 5-a). In this setup, 3 and $4 \mathrm{~mm}$ ice loading were investigated, as well as, interference from a person standing between the reader and antenna, emulating a real-world non-line of sight (NLoS) case. Fig. 14-a shows the variation in $\Delta$ RSS, in addition to the sensing system output $p$.

In Fig. 14-a, it can be observed that the presence of a person between the tag and the reader reduces the RSS, at time $=60 \mathrm{~s}$, where the backscattered signal is shielded by the person. Nevertheless, the ice loading still results in over $7 \mathrm{~dB}$ RSS increase enabling its correct detection as indicated by $p$ on the secondary axis. Furthermore, at time $=220 \mathrm{~s}$ when the person shields the ice-loaded tag, the RSS drop does not result in an incorrect sensor readout, demonstrating the resilience of the proposed sensing approach to interference from nearby humans such as pedestrian in a smart city use case.

A more adverse case is the presence of a metal shield in between the sensing tag and the RFID reader, as shown in Fig. 13-b. Fig. 14-b shows that the metal shadowing halfway between the tag and the reader reduces the RSS by $5 \mathrm{~dB}$, at time $=60 \mathrm{~s}$. Nevertheless, the $11 \mathrm{~dB}$ increase in the measured RSS following ice-loading results in a correct detection, as shown in the system's output $p$. 

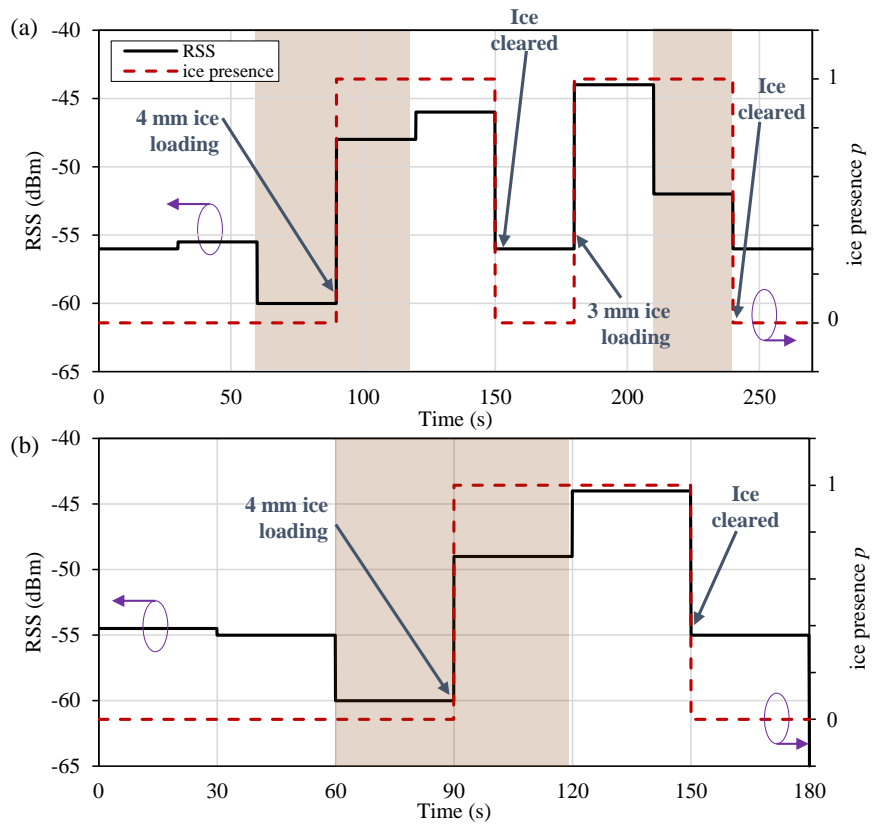

Fig. 14. Measured sensor response at $5 \mathrm{~m}$ for ice loading in the presence and absence of obstructions, indicated by the shaded regions: (a) human obstruction as in Fig. 15-a; (b) metal obstruction as in Fig. 15-b.

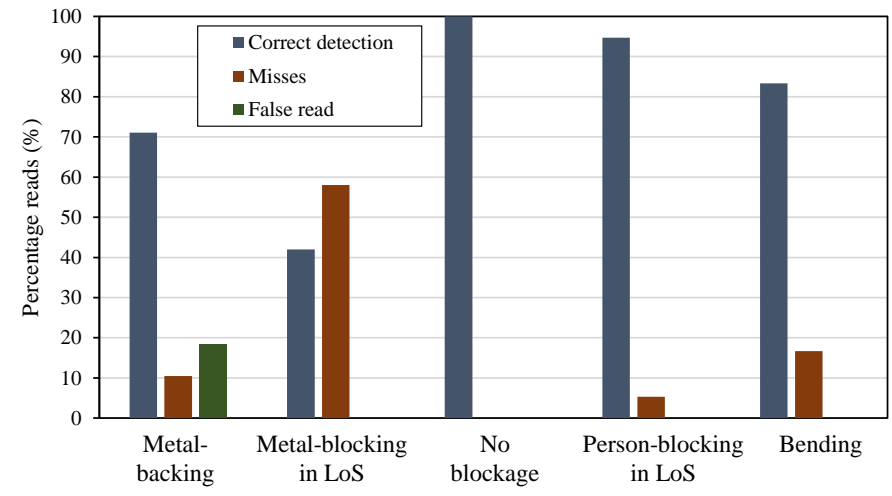

Fig. 15. The measured sensor's output accuracy, in terms of correct, missed, and false ice detection, for different test setups, calculated using (5).

In Fig. 14, the sensor's output has been evaluated for a single separation, $d=5 \mathrm{~m}$. In order to evaluate the percentage accuracy of the proposed sensing system, the distance was swept from 0.5 to $10.5 \mathrm{~m}$, for all the scenarios shown in Fig. 13. In addition, a tag bent around a $7.5 \mathrm{~cm}$ radius was measured, to demonstrate that the tags' flexibility does not affect the sensing performance. The measurements were taken every $0.5 \mathrm{~m}$, and the output has been classified into: (a) correct detection of ice; (b) a missed read, where the ice was not detected; (c) a false match, where an increase in the RSS resulted in a false read. Fig 15 shows the output of the sensor for all test cases investigated.

In the LoS unobstructed case, the sensor can always detect the presence of ice, as shown in Fig. 15 and characterized extensively in Section IV. The lowest detection rate is observed for a metal plate between the reader and the tag (i.e. in LoS), which is anticipated for any RF-based sensing or communication mechanism. For all the distance points between 0.5 and
$10.5 \mathrm{~m}$, only a single point was missed in presence of a person between the tag and the reader, demonstrating the resilience of RFID-based sensing to nearby humans. For the metal-backed tag (Fig. 13-c), the sensing system encounters false matches, where the higher gain of the metal-backed antenna results in a similar RSS change to that introduced by the presence of ice. To explain, a metal plate placed at $25 \mathrm{~cm}$ behind the tag will act as a reflector, typically increasing the gain of the antenna by at least $3 \mathrm{~dB}$. Nevertheless, for tags' backed by metal at more than $40 \mathrm{~cm}$, no influence was observed on the sensors' accuracy, showing their high suitability for for practical deployment. Based on all the scenarios considered for the different distances, the total accuracy of the system is evaluated as

$$
\text { Accuracy }=\frac{|\mathrm{CD}|}{|\mathrm{CD}|+|\mathrm{M}|+|\mathrm{F}|}
$$

where $|\mathrm{CD}|$ is the number of correct detections, $|\mathrm{M}|$ is the number of misses, and $|\mathrm{F}|$ is the number of false matches. The system achieves an $80 \%$ overall accuracy, and $86 \%$ when excluding the results with a metallic obstruction in line of sight.

Finally, while a sensing tag placed directly on a metal plate will not be readable due to detuning, this is due to the antenna design (a dipole antenna) and will manifest in any RFID-based system based on a wire-type antenna. Should the proposed sensing approach be applied to detecting the presence of ice on metals, a microstrip patch antenna with a full ground plane backing can be used [30]. However, as patch antennas do require more area and thickness compared to dipoles, the proposed sensing approach was demonstrated based on a compact dipole, more suited to wide-scale deployment.

\section{B. Comparison with Other Ice Sensing Approaches}

Recently, an optic fibre-based approach was proposed based on the observed distortion of light propagation when ice deposits on a fibre [31]. However, this approach requires the laser source and the photodetector to exist at both ends of the optic, whereas RFID tags, of significantly lower cost, can be deployed in many locations and covered by a single reader with up to $10 \mathrm{~m}$ range. Moreover, a sensing fiber will only cover a small surface area due to its physical size, unlike an RF wireless sensor which can cover a spot with several meters radius as shown in the measured results (Fig. 11). NIR sensors and computer vision can detect ice remotely for road surfaces [15]. However, optical methods were found to be generally less sensitive than microwave sensors [18].

Compared to the recently reported RF ice and frost sensors [18], the proposed antenna-based sensor enables wireless detection of many ice sensors using a single RFID reader, with a measured range of $10 \mathrm{~m}$. However a two-port resonator requires active circuitry to be sampled. In addition, should the two-port resonator in [18] be connected to two tag antennas (i.e. chipless RFID), its read-range will not exceed 1 metre due to the sensor having an $S_{21}<-20 \mathrm{~dB}$, significantly reducing the backscatterd signal. As for heater-integrated sensors [19], [32], both the low frequency and microwave sensors are one or two-port devices which require an active sampling circuit 
for each deployed sensor, adding to the complexity of the sensing approach and limiting their pervasiveness in a city environment.

Commercial RFID "sensing" ICs, such as the temperaturesensing AMS SL900-A, could represent an alternative to the proposed RF-based approach. However, RFID sensing ICs typically have a significantly lower sensitivity, requiring around $-7 \mathrm{dBm}$ to be powered in battery-free mode, whereas most standard Gen-2 RFID tags (such as the NXP UCODE 7 used in this work), require less than $-20 \mathrm{dBm}$, enabling a readrange around $10 \mathrm{~m}$. In addition, RFID sensor ICs are typically larger and more expensive than their passive counterparts, restricting wide-scale deployment. Another RF alternative is utilizing wireless power transmission to a rectenna-powered sensor node. However, state-of-the-art rectennas, based on off-the-shelf components, operating at a similar range to the proposed tag (up to 10 metres) cannot charge a conventional microcontroller in few seconds [33], or produce sufficient voltage without a boost converter [34], [35], resulting in a long delay before the node can be charged to a sufficient level to perform any sensing. Therefore, the proposed RFID-based sensing mechanism is the most suited method for long-range and wide-scale ice detection.

Finally, it is key to note that while the proposed RFID sensor achieves a state-of-the-art wireless read-range compared to most reported RFID sensors, the read-range is limited by the tag's turn-on threshold $(-21 \mathrm{dBm})$ and the reader's sensitivity $(-74 \mathrm{dBm})$. For example, a higher sensitivity reader such as [36] would enable the proposed RFID ice sensor to be interrogated at ranges in excess of $20 \mathrm{~m}$. Furthermore, recent advances in CMOS rectifiers demonstrating high RFDC power conversion efficiencies as low as $-30 \mathrm{dBm}$ [37] will enable future RFID ICs to operate with a much higher sensitivity, where the tag will require less than $-30 \mathrm{dBm}$ of $\mathrm{RF}$ power to report its ID to the reader.

\section{CONCLUSION}

In this paper, a UHF RFID-based approach was proposed for long-range wireless ice detection and monitoring. Despite the low permittivity of ice, the proposed sensor demonstrates that RFID sensing is highly-suited for detecting freezing events, the presence of ice, as well as monitoring the thawing of ice with high linearity. Using sub-100 $\mu$ m-thick RFID tags, the existence of mm-thick layers of ice as a superstrate translates to a resonance shift which can be detected wirelessly through the tag's RSS, even in a highly echoic multi-path environment. The proposed sensor was demonstrated in a variety of usecases showing state-of-the-art performance. The ice-loaded sensor tags maintain read-range of $10 \mathrm{~m}$ with $10 \mathrm{~mm}$ of ice cover and up to $14 \mathrm{~dB}$ RSS sensitivity, with the antenna's measured loaded response showing a close agreement to the simulated results. Based on the performance of the proposed sensor, RFID sensing is one of the most promising candidates for detecting and monitoring ice in a range of smart cities and industrial applications.

\section{REFERENCES}

[1] P. Mezzanotte, V. Palazzi, F. Alimenti, and L. Roselli, "Innovative rfid sensors for internet of things applications," IEEE Journal of Microwaves, vol. 1, no. 1, pp. 55-65, 2021.

[2] C. Occhiuzzi, S. Caizzone, and G. Marrocco, "Passive uhf rfid antennas for sensing applications: Principles, methods, and classifcations," IEEE Antennas and Propagation Magazine, vol. 55, no. 6, pp. 14-34, 2013.

[3] M. S. Rohei, E. Salwana, N. B. A. K. Shah, and A. S. Kakar, "Design and testing of an epidermal rfid mechanism in a smart indoor human tracking system," IEEE Sensors Journal, vol. 21, no. 4, pp. 5476-5486, 2021.

[4] I. Ullah, R. Horne, B. Sanz-Izquierdo, and J. C. Batchelor, "Rfid ac current sensing technique," IEEE Sensors Journal, vol. 20, no. 4, pp. 2197-2204, 2020

[5] M. A. S. Tajin, W. M. Mongan, and K. R. Dandekar, "Passive rfidbased diaper moisture sensor," IEEE Sensors Journal, vol. 21, no. 2 pp. 1665-1674, 2021

[6] R. Gonçalves, S. Rima, R. Magueta, P. Pinho, A. Collado, A. Georgiadis, J. Hester, N. B. Carvalho, and M. M. Tentzeris, "Rfid-based wireless passive sensors utilizing cork materials," IEEE Sensors Journal, vol. 15, no. 12 , pp. 7242-7251, 2015

[7] P. Sen, S. N. R. Kantareddy, R. Bhattacharyya, S. E. Sarma, and J. E. Siegel, "Low-cost diaper wetness detection using hydrogel-based rfid tags," IEEE Sensors Journal, vol. 20, no. 6, pp. 3293-3302, 2020.

[8] Y. He, M. M. Li, G. C. Wan, and M. S. Tong, "A passive and wireless sensor based on rfid antenna for detecting mechanical deformation," IEEE Open Journal of Antennas and Propagation, vol. 1, pp. 426-434, 2020.

[9] V. Palazzi, F. Alimenti, P. Mezzanotte, G. Orecchini, and L. Roselli, "Zero-power, long-range, ultra low-cost harmonic wireless sensors for massively distributed monitoring of cracked walls," in 2017 IEEE MTT-S International Microwave Symposium (IMS), 2017, pp. 1335-1338.

[10] A. J. R. Hillier, V. Makarovaite, C. W. Gourlay, S. J. Holder, and J. C. Batchelor, "A passive uhf rfid dielectric sensor for aqueous electrolytes," IEEE Sensors Journal, vol. 19, no. 14, pp. 5389-5395, 2019.

[11] J. Nummela, L. Ukkonen, and L. Sydänheimo, "Passive uhf rfid tags in arctic environment," vol. 2, no. 1, 2008.

[12] P. Blasco, J. Palacios, and S. Schmitz, "Effect of icing roughness on wind turbine power production," Wind Energy, vol. 20, no. 4, pp. 601617, 2017.

[13] "Frozen pipes cause billions in damage, but can be prevented," Insurance Journal, 2006, Online: $\quad$ https://www.insurancejournal.com/magazines/magfeatures/2006/01/02/64842.htm. [accessed: 12/03/2021.

[14] "Slipping on Ice: Statistics on Injuries," GRITT, 2017, Online: http://info.gritit.com/blog/slipping-on-ice-statistics-oninjuries: :text=1 [accessed: 07/03/2021.

[15] P. Jonsson, J. Casselgren, and B. Thörnberg, "Road surface status classification using spectral analysis of nir camera images," IEEE Sensors Journal, vol. 15, no. 3, pp. 1641-1656, 2015.

[16] N. Najibi, S. Jin, and X. Wu, "Validating the variability of snow accumulation and melting from gps-reflected signals: Forward modeling,' IEEE Transactions on Antennas and Propagation, vol. 63, no. 6, pp. 2646-2654, 2015.

[17] V. Komarov, S. Wang, and J. Tang, Permittivity and Measurements. American Cancer Society, 2005.

[18] B. Wiltshire, K. Mirshahidi, K. Golovin, and M. H. Zarifi, "Robust and sensitive frost and ice detection via planar microwave resonator sensor," Sensors and Actuators B: Chemical, vol. 301, p. 126881, 2019.

[19] R. Kozak, B. D. Wiltshire, M. A. R. Khandoker, K. Golovin, and M. H. Zarifi, "Modified microwave sensor with a patterned ground heater for detection and prevention of ice accumulation," ACS Applied Materials and Interfaces, vol. 12(49), pp. 55 483-55 492, 2020.

[20] R. N. Dean, "A pcb sensor for detecting icing events," IEEE Sensors Letters, vol. 5, no. 1, pp. 1-4, 2021.

[21] M. Wagih, Y. Wei, A. Komolafe, R. Torah, and S. Beeby, "Reliable UHF Long-Range Textile-Integrated RFID Tag Based on a Compact Flexible Antenna Filament," Sensors, vol. 20 (12), p. 3435, 2020.

[22] Yi Huang and Kevin Boyle, Antennas: From Theory to Practice. West Sussex, United Kingdom: John Wiley \& Sons Ltd, 2008.

[23] K. Rao, P. Nikitin, and S. Lam, "Antenna design for uhf rfid tags: a review and a practical application," IEEE Transactions on Antennas and Propagation, vol. 53, no. 12, pp. 3870-3876, 2005.

[24] G. Marrocco, "The art of UHF RFID antenna design: impedancematching and size-reduction techniques," IEEE Antennas Propag. Magazine, vol. 50, 1, pp. $66-79,2008$. 
[25] J. Virtanen, L. Ukkonen, T. Bjorninen, A. Z. Elsherbeni, and L. Sydänheimo, "Inkjet-printed humidity sensor for passive uhf rfid systems," IEEE Transactions on Instrumentation and Measurement, vol. 60, no. 8, pp. 2768-2777, 2011.

[26] K. Palmer and M. van Rooyen, "Simple broadband measurements of balanced loads using a network analyzer," IEE Transactions on Instrumentation and Measurements, vol. 55, no. 1, pp. 266 - 272, 2006.

[27] M. Wagih, G. S. Hilton, A. S. Weddell, and S. Beeby, "Dual-Band DualMode Textile Antenna/Rectenna for Simultaneous Wireless Information and Power Transfer (SWIPT)," IEEE Trans. Antennas Propag., 2021.

[28] S. Tsujita, N. Sakai, K. Itoh, and S. Tsuchimoto, "920mhz band high sensitive rectenna with a small loop antenna," in 2019 IEEE Asia-Pacific Microwave Conference (APMC), 2019, pp. 1274-1276.

[29] D. Gomez, M. Sroka, and J. L. G. G. Jimenez, "Process and Temperature Compensation for RF Low-Noise Amplifiers and Mixers," IEEE Trans. Circuits And Systems, vol. 57 no. 6, pp. 1204 - 1211, 2010.

[30] S.-L. Chen and K.-H. Lin, "A slim rfid tag antenna design for metallic object applications," IEEE Antennas and Wireless Propagation Letters, vol. 7, pp. 729-732, 2008.

[31] Y. H. Tai, A. S. A. Kamal, Y. J. Park, P. C. Tsai, Y. L. Ho, P. K. Wei, H. Daiguji, and J. J. Delaunay, "Real-time monitoring of frost/defrost processes using a tapered optical fiber," IEEE Sensors Journal, vol. 21 , no. 5, pp. 6188-6194, 2021.

[32] M. Knoll, C. Offenzeller, B. Jakoby, and W. Hilber, "Embedded temperature and anti-icing monitoring systems directly printed on 3d shaped substrates," IEEE Sensors Journal, vol. 20, no. 10, pp. 5314-5321, 2020.

[33] M. Wagih, N. Hillier, S. Yong, A. S. Weddell, and S. Beeby, "Rfpowered wearable energy harvesting and storage module based on etextile coplanar waveguide rectenna and supercapacitor," IEEE Open Journal of Antennas and Propagation, vol. 2, pp. 302 - 314, 2021.

[34] M. Wagih, A. S. Weddell, and S. Beeby, "High-Efficiency Sub-1 GHz Flexible Compact Rectenna based on Parametric Antenna-Rectifier CoDesign," in 2020 IEEE/MTT-S International Microwave Symposium (IMS), 2020

[35] V. Palazzi, J. Hester, J. Bito, F. Alimenti, C. Kalialakis, A. Collado, P. Mezzanotte, A. Georgiadis, L. Roselli, and M. M. Tentzeris, "A Novel Ultra-Lightweight Multiband Rectenna on Paper for RF Energy Harvesting in the Next Generation LTE Bands," IEEE Trans. Microw. Theory Techn., vol. 66 no. 1, pp. $366-379,2018$.

[36] H. Min, "High Sensitivity RFID for Tracking Assets," GS1: The global language of business, pp. 2016, Online: https://www.gs1.org/sites/default/files/10_--_2016_brussels_event_ fudan_university_long_range_rfid_final.pdf [accessed: 12/03/2021.

[37] A. S. Almansouri, J. Kosel, and K. N. Salama, "A Dual-Mode Nested Rectifier for Ambient Wireless Powering in CMOS Technology," IEEE Trans. Microw. Theory Techn., vol. 68 no. 5, pp. 1754 - 1762, 2016.

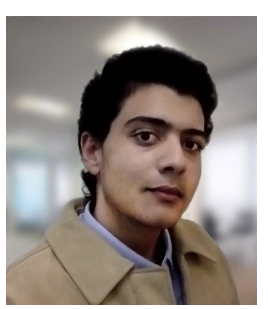

Mahmoud Wagih (GS'18, M'21) received his B.Eng. (Hons.) from the University of Southampton in September 2018, and his Ph.D. in April 2021.

In 2017 he worked as a Research Assistant at the University of Southampton. In 2018, he was a Hardware Engineering Intern at Arm, and, in 2020, a Research Intern at Arm, Cambridge, U.K. He is currently a Senior Research Assistant at the University of Southampton leading a Flexible Innovation Fund project on Green RF Sensing. His interests lie in RF energy harvesting, wearable antennas, and RFID sensing. He has 14 journal and 23 conference publications, and has delivered several invited webinars on these topics.

Dr. Wagih is a member of the Institute of Engineering and Technology (MIET). He was the recipient of the Best Undergraduate Project Prize at the University of Southampton, in 2018, and was selected for the IEEE International Microwave Symposium Project Connect, 2019. He received the Best Student Paper Award at the IEEE Wireless Power Transfer Conference, 2019, and the Best Oral Paper at PowerMEMS, 2019. He won the Best 3MT Presentation Prize (second place) at the IEEE Microwave Week, 2020, and was a session co-chair at the 15th European Conference on Antennas and Propagation EuCAP, 2021. He has acted as a reviewer for 9 journals including the IEEE TRansactions on ANTENnas and PRopagation, IEEE Sensors Letters, Sensors and ACtuators A: Physical (Elsevier), and SENSORS.

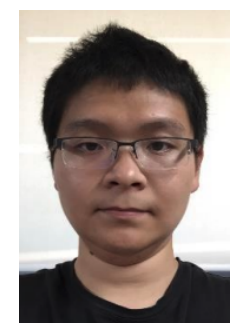

Junjie Shi received a B.Sc. degree from the Nanjing Institute of Technology, China in 2008, an M.Sc. degree in Microelectromechanical systems in 2012, and a Ph.D. in 2017 at the University of Southampton.

He was appointed as a Research Fellow in the School of Electronics and Computer Science (ECS) in 2017. His research interest covers a board range of wearables including but not limited to sensors, actuators, memory, and energy harvesting etc. As well as those wearable devices, he is interested in the fabrication technologies, such as 3D printing, dispenser printing, inkjet printing, micro-fabrications, which enable conventional and novel devices to be directly fabricated on to textiles. 